\title{
Hypolipidemic Effects of Alkaloids from Rhizoma Coptidis in Diet-Induced Hyperlipidemic Hamsters
}

Authors

Affiliations
Kai He ${ }^{1,2}$, Shuming Kou ${ }^{3}$, Zongyao Zou ${ }^{1}$, Yinran $\mathrm{Hu}^{1}$, Min Feng ${ }^{1,4}$, Bing Han ${ }^{3}$, Xuegang $\mathrm{Li}^{1}$, Xiaoli Ye ${ }^{3}$

${ }^{1}$ Chongqing Productivity Promotion Center for the Modernization of Chinese Traditional Medicine, School of Pharmaceutical Sciences, Southwest University, Chongqing, China

${ }^{2}$ Department of Clinical Laboratory, Hunan University of Medicine, Hunan, China

${ }^{3}$ School of Life Sciences, Southwest University, Chongqing, China

${ }^{4}$ College of Environmental and Biological Engineering, Chongqing Technology and Business University, Chongqing, China
Key words

- Rhizoma Coptidis

- Ranunculaceae

- alkaloids

- hyperlipidemic hamsters

- antihyperlipidemic

- mechanism received March 4, 2015

revised Dec. 16, 2015

accepted Dec. 17, 2015

\section{Bibliography}

Dol http://dx.doi.org/

10.1055/s-0035-1568261

Published online February 5 , 2016

Planta Med 2016; 82: 690-697

(c) Georg Thieme Verlag KG

Stuttgart - New York ·

ISSN 0032-0943

\section{Correspondence}

Xuegang Li

Chongqing Productivity Promotion Center for the Modernization of Chinese Traditional Medicine School of Pharmaceutical Sciences

Southwest University No. 2 Tiansheng road Chongqing 400716

China

Phone: +8602368250728

Fax: + 8602368250728

xuegangli@swu.edu.cn

Correspondence

Xiaoli Ye

School of Life Sciences

Southwest University

No. 2 Tiansheng road

Chongqing 400715

China

Phone: +8602368250728

Fax: + 8602368250728

yexiaoli@swu.edu.cn

\section{Abstract}

This study was conducted to evaluate the antihyperlipidemic activity of five major alkaloids in Rhizoma Coptidis using high-fat- and high-cholesterol-induced hyperlipidemic hamsters. Hyperlipidemic hamsters were treated with coptisine, berberine, jatrorrhizine, palmatine, epiberberine, and total Rhizoma Coptidis alkaloids with a dose of $46.7 \mathrm{mg} / \mathrm{kg} \times$ day for 140 days. Serum total cholesterol, triglyceride, high-density lipoprotein cholesterol, low-density lipoprotein cholesterol, and total bile acids were examined after alkaloid treatment. The results showed that all therapy agents prevented body weight gain, reduced the serum total cholesterol, and increased the high-density lipoprotein cholesterol of hamsters. Berberine, jatrorrhizine, and total Rhizoma Coptidis alkaloids decreased the triglyceride level in hyperlipidemic hamsters, while coptisine, jatrorrhizine, palmatine, and total Rhizoma Coptidis alkaloids significantly suppressed the elevation of the low-density lipoprotein cholesterol level. The fecal excretion of bile acids was significantly elevated by berberine, coptisine, jatrorrhizine, palmatine, total Rhizoma Coptidis alkaloids, and orlistat. Notably, total Rhizoma Coptidis alkaloids possess a much stronger lipid-lowering effect than the pure Rhizoma Coptidis alkaloids. Quantitative reverse transcription-polymerase chain reaction analyses revealed that Rhizoma Coptidis alkaloids could retard the synthesis of cholesterol by downregulating the mRNA expression of 3-hydroxy-3-methyl glutaryl coenzyme A

\section{Introduction}

$\nabla$

Over the last decades, there has been a significant increase in obesity in the general population of developed countries and a growing concern in the developing world as well. According to a sur- reductase and accelerate the clearance of lipids by upregulating the low-density lipoprotein receptor, cholesterol $7 \alpha$-hydroxylase, and uncoupling protein-2 expression. These findings highlight the critical role of Rhizoma Coptidis alkaloids in hyperlipidemia treatment. Thus, they need to be considered in future therapeutic approaches.

\section{Abbreviations \\ $\nabla$}

ASBT: apical sodium-dependent bile salt transporter

CYP7A1: cholesterol $7 \alpha$-hydroxylase

DM: diabetes mellitus

FGF: fibroblast growth factor

HDL: high-density lipoprotein

HFHC: high fat and high cholesterol

HMGCR: 3-hydroxy-3-methyl glutaryl coenzyme A reductase

LDL: low-density lipoprotein

LDLR: low-density lipoprotein receptor

NC: $\quad$ normal control group

qRT-PCR: quantitative reverse transcription PCR RC: $\quad$ Rhizoma Coptidis

TBA: total bile acids

TC: $\quad$ total cholesterol

TG: triglyceride

TRCA: total Rhizoma Coptidis alkaloids

UCP-2: uncoupling protein-2

Supporting information available online at http://www.thieme-connect.de/products

vey published in 2010, there were 325 million obese individuals in China and this number will continue to increase [1]. Obesity is the main cause of dyslipidemia, which is associated with a higher risk of developing diabetes and cardiovascular disease [2]. Dyslipidemia is characterized by in- 
creased total cholesterol, triglyceride, and LDL levels, and also by declined HDL levels $[3,4]$. Controlling lipid levels can effectively interfere with the progression of atherosclerosis and reduce cardiovascular events [5]. Currently, amfepramone and simvastatin were widely used for the treatment of obesity. But these drugs have several adverse effects [6]. Therefore, the development of safe and efficient lipid-lowing drugs has attracted considerable research interest.

As early as Anno Domini 220, Rhizoma Coptidis (Coptis chinensis Franch, Ranunculaceae) was used in patients suffering from DM. Clinical studies have revealed that the plasma TG levels of type 2 diabetes mellitus patients were substantially increased, whereas HDL-C levels were markedly reduced in both men and women compared with the nondiabetic controls [7]. The lipid changes associated with DM were attributed to insulin resistance and were considered the major risk factors for cardiovascular disease in DM. Thus, the beneficial effects of RC on DM patients are probably related to the lipids regulation role of RC alkaloids [8]. In fact, berberine isolated from RC could upregulate LDLR expression via activating the extracellular signal-regulated kinase pathway and reducing serum cholesterol, TG, and LDL cholesterol in hypercholesterolemic patients [9]. Recent studies have suggested that berberine treatment significantly decreased blood lipid levels both in obese subjects and in Sprague Dawley rats [10-12]. Moreover, meta-analysis of clinical trials have revealed that the administration of berberine produced a significant reduction in total cholesterol, triglycerides, and LDL cholesterol levels, with a remarkable increase in HDL [13]. Nevertheless, little attention has been given to investigating the lipid-lowering effects of other RC alkaloids including coptisine, jatrorrhizine, palmatine, and epiberberine, and the underlying antihyperlipidemic mechanisms remain largely unknown.

The dynamic equilibrium of lipids in the body is subtly regulated by several key enzymes such as LDLR, HMGCR, CYP7A1, etc. The dietary modulation of cholesterol absorption of Syrian golden hamsters is similar to that of humans and it has been demonstrated that the lipoprotein profile of hamsters resembles that of humans fed an HFHC diet [14]. This study was therefore undertaken to investigate the antihyperlipidemic effects and mechanisms of pure RC alkaloids (coptisine, berberine, jatrorrhizine, palmatine, epiberberine; $\boldsymbol{O}$ Fig. 1) and TRCA in hamsters. It is worth noting that TRCA exhibited the strongest weight-lowering effect in HFHC-induced hyperlipidemic hamsters compared with the pure RC alkaloid.

\section{Results}

$\nabla$

The concentration of each RC alkaloid was calculated from the peak areas using the normalization method. As shown in O Fig. 2, the alkaloids in the RC extract mainly include jatrorrhizine, epiberberine, coptisine, palmatine, and berberine, and the content of these alkaloids were $11.0 \%, 8.0 \%, 9.8 \%, 9.0 \%$, and $47.0 \%$, respectively.

The hyperlipidemic hamsters, weighing approximately $135 \mathrm{~g}$, were obtained after 8 weeks of being fed an HFHC diet. As shown in 0 Fig. 3, after 105 days of treatment, the body weight of each hamster reached its highest value, then gradually decreased, and finally returned to a stable value (see Supporting Information). Nevertheless, the body weight gain of the HFHC group was the largest, followed by the normal group, jatrorrhizine, coptisine, berberine, palmatine, epiberberine, orlistat, and TRCA ( $\odot$ Ta-

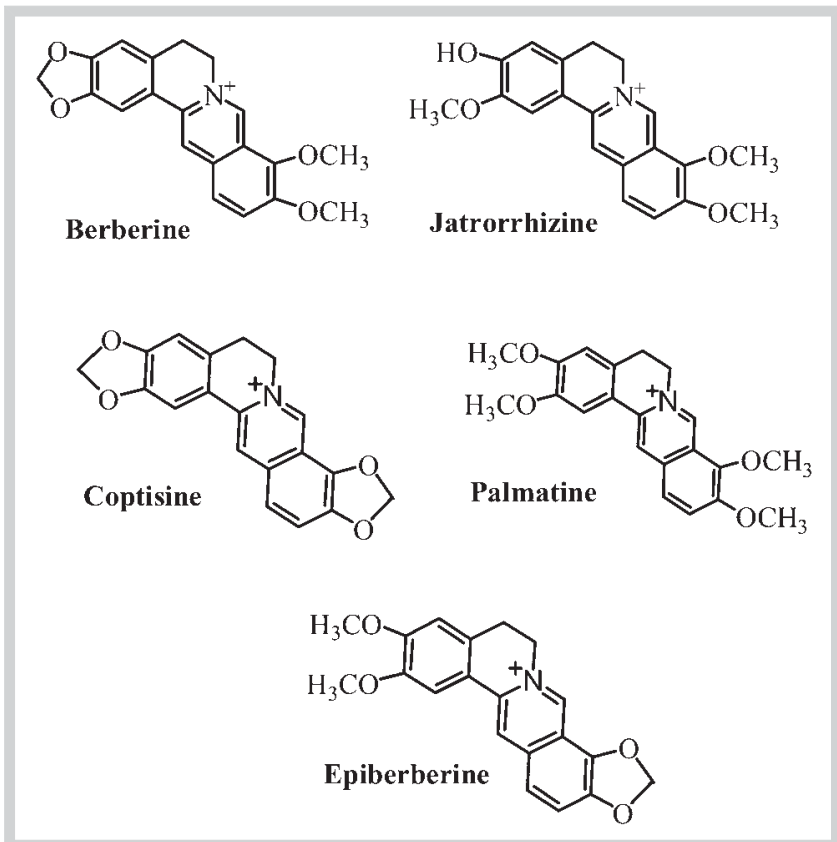

Fig. 1 The structure of five major alkaloids in Rhizoma Coptidis.

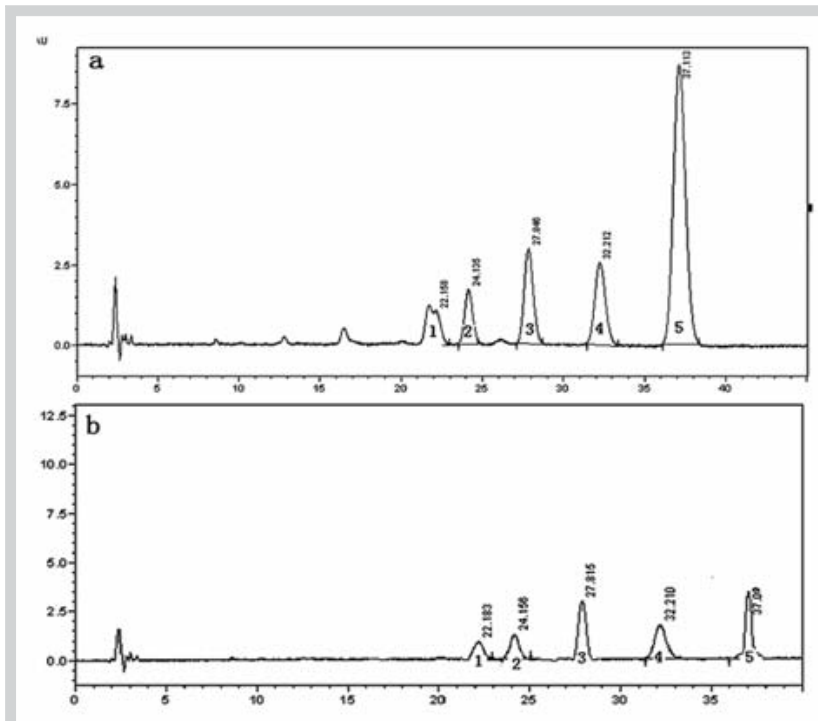

Fig. 2 (a) Chromatogram of crude alkaloids in Rhizoma Coptidis extracts (b) chromatogram of standard compounds. 1. jatrorrhizine; 2. epiberberine; 3 . coptisine; 4 . palmatine; 5 . berberine.

ble 1). After 120 days of treatment, animals supplemented with jatrorrhizine, coptisine, berberine, palmatine, epiberberine, orlistat, and TRCA weighed 6.3\%, 8.2\%, 9.1\%, 10.5\%, 11.2\%, $12.2 \%$, and $17.3 \%$ less than the HFHC group, respectively. These data suggested that both RC alkaloids and TRCA could slow down the weight gain of hamsters induced by an HFHC diet. Notably, TRCA exhibited the strongest weight-lowering effect compared with each pure RC alkaloid and orlistat.

The relative liver weight was not significantly different among groups, but the epididymal adipose weight of the drug-treated group was significantly different when compared to the HFHC 


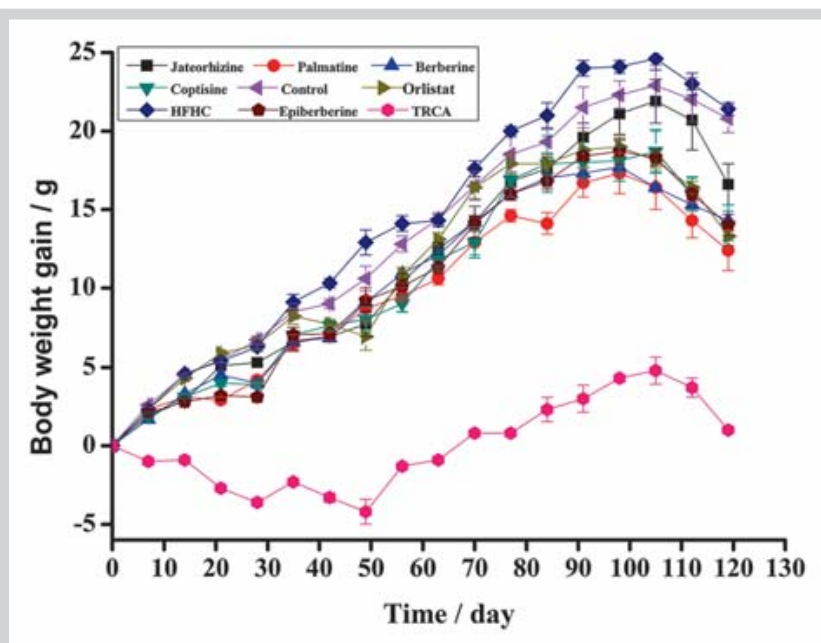

Fig. 3 The body weight gain in all studied hamsters groups. The body weight gain of HFHC group is the largest, followed by normal group, jatrorrhizine, coptisine, berberine, palmatine, epiberberine, orlistat and TRCA. Values were means $\pm S D, n=6$ hamsters in each group. (Color figure available online only.)

group ( $\mathrm{p}<0.05$ ). As shown in $\bigcirc$ Table 1, HFHC induced a higher epididymal adipose weight. Pure RC alkaloids, TRCA, and orlistat prevented the accumulation of lipids in the epididymal adipose of hamsters. The relative kidney weight (g/100 g body weight) in the HFHC group was lower than normal group, because hyperlipidemic hamsters accumulated more lipids in adipose tissue. Although there is no significant difference, the administration of TRCA, epiberberine, palmatine, and orlistat improved the relative kidney weight compared with the HFHC group.

It has been demonstrated that a high triglyceride level in the plasma is a risk factor for cardiovascular disease independent of the high-density lipoprotein cholesterol level [15]. Besides, excessive triglycerides coincide with a high ratio of plasma LDL cholesterol, which will lead to impaired vascular reactivity [16]. In this study, an HFHC diet led to a significant increase in TC (74.7\%), TG (149.0\%), LDL-C (44.7\%), and TBA (166.0\%) in the serum of the hamsters compared with the NC. Feeding hamsters with both pure RC alkaloids and TRCA displayed a significantly lower serum TC compared to the HFHC group ( Table 2 ), while only orlistat, berberine, jatrorrhizine, epiberberine, and TRCA decreased the TG level. The HDL-C values of hamsters fed pure RC alkaloids significantly increased compared with the HFHC group. Coptisine, jatrorrhizine, palmatine, berberine, and TRCA significantly suppressed the elevation of the LDL-C level in hyperlipidemic hamsters. Considering all RC alkaloids and TRCA showed an excellent weight-lowering effect in hyperlipidemic hamsters, we speculate that the weight-lowering effect of RC alkaloids could be attributed to their impact on serum lipids. HFHC results in a higher TBA level in the serum of hamsters. Orlistat, berberine, and TRCA exhibited a serum TBA-lowering effect compared with the HFHC group. These results demonstrated the beneficial effects of $\mathrm{RC}$ alkaloids on serum lipid profiles in hyperlipidemic animals. As the body weight is significantly correlated with the serum lipids, the authors investigated whether the lipid-lowering effects were achieved by lowering the body weight or were direct effects of the treatment compounds. An ANCOVA indicated that the TG levels in the hamsters were significantly affected by body weight $(\mathrm{p}=0.04)$, while there is no interaction between TC, LDLC, HDLC, and TBA values and body weight change $(\mathrm{p}=0.59,0.26,0.44,0.09$, respectively). Therefore, the lipid-lowering effects of $\mathrm{RC}$ alkaloids were mainly achieved by mediating the lipid metabolism in hyperlipidemic hamsters rather than reducing body weight.

To determine the effects of RC alkaloids on the excretion and conversion of cholesterol, the feces of the hamsters were collected 3 days before the experiment and the 3 days prior to the termination of the study. As shown in Table 3, there was no obvious changes in the dry weight of hamster feces among all groups. The HFHC diet caused a significant increase in excreted cholesterol and TBA in the feces. RC alkaloid administration raised the level of cholesterol and TBA in the feces when compared to the HFHC group. It is well acknowledged that the fecal excretion of bile acids can be elevated by dietary cholesterol. In this study, all RC alkaloids and TRCA significantly enhanced the fecal excretion of bile acids. Meanwhile, the fetal cholesterol levels in the coptisine, jatrorrhizine, palmatine, berberine, TRCA, and orlistat groups were increased by $27.0 \%, 12.1 \%, 21.8 \%, 11.6 \%, 12.5 \%$ and $14.5 \%$, respectively, compared to the HFHC group. These results suggested that both pure RC alkaloids and TRCA could promote the conversion of cholesterol into bile acids as well as accelerate the fecal excretion of bile acids.

To explore the molecular mechanisms for the antihyperlipidemic efficiency of RC alkaloids, the mRNA expression of lipid metabolism-related genes was investigated by qRT-PCR. As shown in - Fig. 4, orlistat, berberine, and palmatine showed significant inhibition of HMGCR $(p<0.01)$. Oral administration of coptisine and TRCA also obviously suppressed the mRNA expression of HMGCR. Additionally, the CYP7A1 mRNA level was significantly increased by $38.4 \%$ and $53.8 \%$ with coptisine, and palmatine therapy, respectively, compared to the HFHC group $(\mathrm{p}<0.01)$. The LDLR mRNA levels increased 3.1-, 3.0-, and 1.9-fold in berberine, jatrorrhizine, and palmatine supplemented groups, respectively, compared to the HFHC group ( $p<0.01$ ). Coptisine and TRCA also enhanced the LDLR mRNA expression in hamsters. HFHC administration significantly increased UCP-2 expression in comparison with the normal group. Among the tested treatments, berberine, palmatine, and TRCA administration elevated the UCP-2 mRNA expression by $20.1 \%, 35.4 \%, 31.0 \%$, respectively. These results suggested that pure RC alkaloids and TRCA could retard the synthesis of cholesterol by downregulating the mRNA expression of HMGCR and accelerate lipid metabolism by upregulating LDLR, CYP7A1, and UCP-2 mRNA expression in the liver of hyperlipidemic hamsters.

ELISAs are characterized by high selectivity as well as low limits of detection. In this study, ELISA kits were applied to the quantitative analysis of HMGCR, CYP7A1, and LDLR in the liver of hamsters. The HMGCR protein level in hamsters was found to decrease and this effect was promoted by RC alkaloid treatment. In accordance with the change in HMCCR mRNA expression, orlistat, coptisine, berberine, palmatine, and TRCA significantly reduced the protein levels of HMCCR by $12.00 \%, 8.24 \%, 6.70 \%, 9.61 \%$, and $4.01 \%$, respectively, compared with the HFHC group. CYP7A1 is the initial and rate-limiting enzyme involved in the conversion of cholesterol to bile acids. As can be seen from $\bullet$ Fig. 5 , the elevated level of CYP7A1 in the liver is possibly a reflection of increased circulation of bile acids in hamsters resulting from HFHC administration. In response to all pure RC alkaloids and TRCA, hamsters exhibited a higher level of CYP7A1, which promoted the conversion of cholesterol to bile acids and led to a depletion of the intrahepatic pool of cholesterol ( Table 3). Among the tested samples, coptisine showed the highest CYP7A1 upregulat- 
Table 1 The body weight and relative organ weight change of hamsters.

\begin{tabular}{|c|c|c|c|c|c|c|c|c|c|}
\hline Treatment & Control & HFHC & Orlistat & TRCA & Coptisine & Epiberberine & Berberine & Palmatine & Jateorhizine \\
\hline Initial body weight (g) & $134.72 \pm 4.10$ & $134.00 \pm 5.73$ & $133.13 \pm 3.62$ & $137.57 \pm 5.00$ & $138.08 \pm 3.78$ & $131.63 \pm 4.42$ & $139.88 \pm 5.91$ & $132.59 \pm 6.28$ & $135.02 \pm 6.08$ \\
\hline Final body weight (g) & $151.71 \pm 6.22^{b}$ & $162.02 \pm 7.75^{a}$ & $142.28 \pm 5.47^{c}$ & $=133.91 \pm 7.08^{d}$ & $148.69 \pm 8.04^{b, c}$ & c $143.76 \pm 3.19^{c}$ & $151.28 \pm 4.40^{b}$ & $145.03 \pm 5.17^{c, d}$ & d 147.10 \\
\hline $\begin{array}{l}\text { Liver }(\mathrm{g} / 100 \mathrm{~g} \\
\text { body weight) }\end{array}$ & $3.08 \pm 0.30$ & $3.29 \pm 0.38$ & $2.92 \pm 0.17$ & $3.17 \pm 0.50$ & $3.33 \pm 0.42$ & $2.88 \pm 0.21$ & $3.19 \pm 0.23$ & $3.16 \pm 0.33$ & $3.33 \pm 0.37$ \\
\hline $\begin{array}{l}\text { Kidney weight } \\
\text { (g/100 g body weight) }\end{array}$ & 08 & \pm 0.06 & 09 & 07 & 09 & 07 & 08 & .05 & 0.06 \\
\hline $\begin{array}{l}\text { Epididymal adipose } \\
\text { (g/100 g body weight) }\end{array}$ & $.43 \pm 0.48^{b}$ & $3.16 \pm 0.36^{a}$ & $2.32 \pm 0.42^{\mathrm{b}}$ & , c $\quad 2.04 \pm 0.25^{b}$, & b,c $\quad 2.33 \pm 0.47^{b, c}$ & c $\quad 2.57 \pm 0.70^{b, c}$ & $2.84 \pm 0.58^{\mathrm{a}, \mathrm{b}}$ & , b $2.53 \pm 0.61^{b, c}$ & c $\quad 2.49 \pm 0.55^{b, c}$ \\
\hline
\end{tabular}

Values are means $\pm S D, n=6$ hamsters in each group. Here, the multiple comparison results were presented using different letters, values with the same letter were not significantly different, whereas, different letters indicate statistically significant differences among groups at $\mathrm{p}<0.05$.

Table 2 Effect of RC alkaloids on serum lipid profile in hyperlipidemic hamsters.

\begin{tabular}{|c|c|c|c|c|c|}
\hline \multirow[t]{2}{*}{ Group } & \multicolumn{5}{|c|}{ Serum lipid profile } \\
\hline & TC (mmol/L) & TG (mmol/L) & LDL-C (mmol/L) & HDL-C (mmol/L) & TBA ( $\mu \mathrm{mol} / \mathrm{L})$ \\
\hline NC & $4.71 \pm 0.41$ & $0.93 \pm 0.04$ & $1.90 \pm 0.07$ & $1.94 \pm 0.11$ & $22.17 \pm 0.61$ \\
\hline HFHC & $8.32 \pm 0.72^{\triangle \Delta}$ & $2.32 \pm 0.20^{\Delta \Delta}$ & $2.75 \pm 0.34^{\Delta \Delta}$ & $2.11 \pm 0.30^{\Delta}$ & $58.82 \pm 3.60^{\Delta \Delta}$ \\
\hline Orlistat & $6.61 \pm 0.42^{* *}$ & $1.92 \pm 0.27^{*}$ & $2.56 \pm 0.15$ & $2.18 \pm 0.12$ & $35.90 \pm 2.01 * *$ \\
\hline Coptisine & $6.60 \pm 0.37^{* *}$ & $2.25 \pm 0.23$ & $2.20 \pm 0.17^{* *}$ & $2.52 \pm 0.19^{* *}$ & $57.94 \pm 4.25$ \\
\hline Berberine & $6.74 \pm 0.56^{*}$ & $2.01 \pm 0.27^{*}$ & $2.34 \pm 0.26^{*}$ & $2.38 \pm 0.11^{*}$ & $48.18 \pm 6.05^{*}$ \\
\hline Jatrorrhizine & $6.51 \pm 0.22^{* *}$ & $1.47 \pm 0.11^{* *}$ & $2.17 \pm 0.18^{* *}$ & $2.34 \pm 0.10^{*}$ & $53.89 \pm 4.96$ \\
\hline Palmatine & $6.16 \pm 0.34^{* *}$ & $2.24 \pm 0.13$ & $2.26 \pm 0.23^{*}$ & $3.44 \pm 0.37^{* *}$ & $55.52 \pm 3.44$ \\
\hline Epiberberine & $6.92 \pm 0.41^{*}$ & $2.03 \pm 0.33^{*}$ & $2.46 \pm 0.15$ & $2.62 \pm 0.27^{* *}$ & $54.40 \pm 3.10$ \\
\hline TRCA & $6.96 \pm 0.88^{*}$ & $1.50 \pm 0.20 * *$ & $2.10 \pm 0.31^{* *}$ & $2.30 \pm 0.11$ & $43.20 \pm 4.00 * *$ \\
\hline
\end{tabular}

Values are means $\pm S D, n=6$ hamsters in each group. TC: total cholesterol; TG: triglyceride; LDL-C: low-density lipoprotein cholesterol; HDL: high-density lipoprotein cholesterol; TBA: total bile acids; ${ }^{\Delta} \mathrm{p}<0.05,{ }^{\Delta \Delta} \mathrm{p}<0.01$ vs. normal control; ${ }^{*} \mathrm{p}<0.05,{ }^{*}{ }^{*} \mathrm{p}<0.01$ vs. HFHC

Table 3 Effect of pure RC alkaloids and TRCA on total cholesterol and bile acids in the feces of hyperlipidemic hamsters.

\begin{tabular}{|c|c|c|c|c|c|c|}
\hline \multirow[t]{2}{*}{ Group } & \multicolumn{2}{|c|}{ Dry weight (g/d) } & \multicolumn{2}{|c|}{ Cholesterol $(\mu \mathrm{mol} / \mathrm{g})$} & \multicolumn{2}{|c|}{ Total bile acids $(\mu \mathrm{mol} / \mathrm{g})$} \\
\hline & $4 W$ & $14 W$ & $4 W$ & $14 W$ & $4 W$ & $14 W$ \\
\hline NC & $1.17 \pm 0.01$ & $1.15 \pm 0.03$ & $1.81 \pm 0.04$ & $1.83 \pm 0.02$ & $0.46 \pm 0.00$ & $0.47 \pm 0.02$ \\
\hline HFHC & $1.17 \pm 0.03$ & $1.18 \pm 0.04$ & $2.45 \pm 0.03^{\Delta \Delta}$ & $2.48 \pm 0.12^{\Delta \Delta}$ & $0.57 \pm 0.01^{\Delta \Delta}$ & $0.56 \pm 0.02^{\Delta \Delta}$ \\
\hline Orlistat & $1.16 \pm 0.03$ & $1.17 \pm 0.02$ & $2.44 \pm 0.02$ & $2.84 \pm 0.08^{*}$ & $0.56 \pm 0.01$ & $0.58 \pm 0.04$ \\
\hline Berberine & $1.17 \pm 0.03$ & $1.18 \pm 0.01$ & $2.49 \pm 0.07$ & $2.77 \pm 0.07^{*}$ & $0.57 \pm 0.02$ & $0.96 \pm 0.07^{* *}$ \\
\hline Coptisine & $1.16 \pm 0.05$ & $1.14 \pm 0.10$ & $2.48 \pm 0.16$ & $3.15 \pm 0.22^{* *}$ & $0.54 \pm 0.16$ & $1.14 \pm 0.21^{* *}$ \\
\hline Jatrorrhizine & $1.16 \pm 0.04$ & $1.15 \pm 0.03$ & $2.41 \pm 0.04$ & $2.78 \pm 0.11^{*}$ & $0.55 \pm 0.03$ & $1.08 \pm 0.04^{* *}$ \\
\hline Palmatine & $1.15 \pm 0.02$ & $1.16 \pm 0.01$ & $2.42 \pm 0.05$ & $3.02 \pm 0.10^{* *}$ & $0.54 \pm 0.04$ & $1.10 \pm 0.14^{* *}$ \\
\hline Epiberberine & $1.16 \pm 0.08$ & $1.15 \pm 0.10$ & $2.41 \pm 0.07$ & $2.58 \pm 0.06$ & $0.56 \pm 0.20$ & $0.91 \pm 0.11^{* *}$ \\
\hline TRCA & $1.16 \pm 0.05$ & $1.15 \pm 0.13$ & $2.44 \pm 0.51$ & $2.79 \pm 0.49^{*}$ & $0.56 \pm 0.32$ & $1.09 \pm 0.10^{* *}$ \\
\hline
\end{tabular}

Values are means $\pm S D, n=6$ hamsters in each group. RC alkaloids administration raised the level of cholesterol and TBA in the feces when compared with the HFHC group. ${ }^{\Delta \Delta} \mathrm{p}<0.01$ vs. normal control; ${ }^{*} \mathrm{p}<0.05,{ }^{* *} \mathrm{p}<0.01$ vs. HFHC

ing activity, followed by palmatine, TRCA, jatrorrhizine, epiberberine, and berberine (increased by $17.01 \%, 15.10 \%, 11.32 \%$, $9.43 \%, 7.55 \%$, and $5.81 \%$, respectively). LDLR was an important receptor involved in the endocytosis of cholesterol. As shown in - Fig. 5, HFHC led to a lower content of LDLR in the hamsters. Except for epiberberine, the LDLR protein levels were greatly increased after coptisine, berberine, jatrorrhizine, palmatine, and TRCA treatment (increased by $11.49 \%, 22.09 \%, 30.08 \%, 22.14 \%$, and $11.52 \%$, respectively) compared to the HFHC group $(\mathrm{p}<0.05)$.

\section{Discussion}

Currently, the rapid development of the world economy has resulted in a great variation in diet composition of most people in developing countries, that is, the shift from staple crops to more flesh (mainly pork in China). High-fat diets are associated with the development of chronic diseases such as Alzheimer's disease, diabetes and hyperlipidemia [17]. Therefore, harmonious regulation of the synthesis and metabolism of cholesterol is crucial to reduce the risk of these cardiovascular diseases.

The synthesis and metabolism of cholesterol of Syrian golden hamsters was similar to that of humans, therefore this animal model was used to evaluate the lipid-lowering effects of RC alkaloids. The body weight gain of all hamsters was consistent with the previous study [18]. Additionally, all therapy agents reduced the serum TC and increased HDL-C values. Berberine, jatrorrhizine, and TRCA decreased the TG level in hyperlipidemic hamsters, while coptisine, jatrorrhizine, palmatine, and TRCA significantly suppressed the elevation of the LDL-C level.

The cholesterol level in the body is subtly regulated by the excretion via conversion to bile acids in the liver as well as the synthe- 


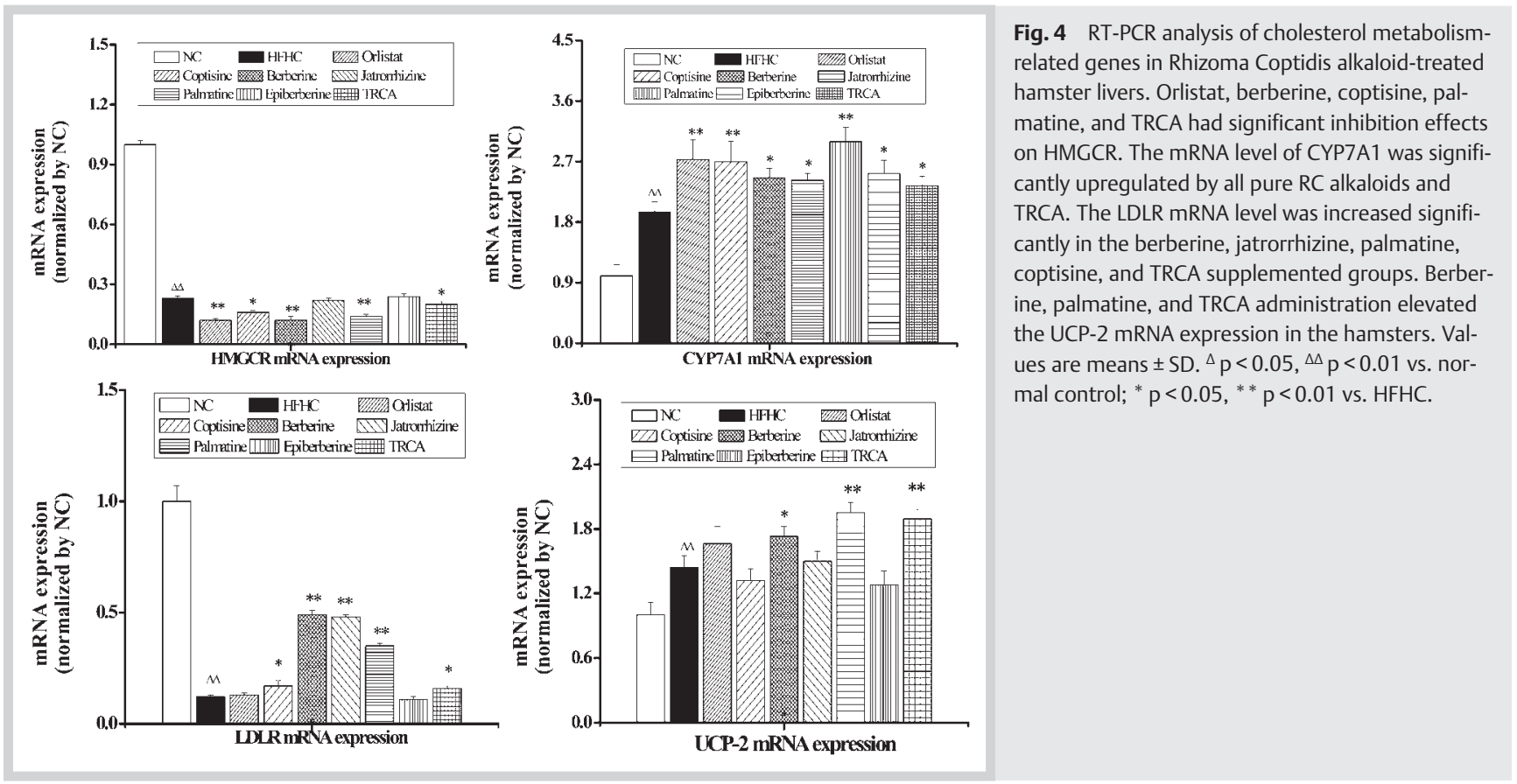

sis and metabolism of cholesterol. The regulation of HMGCR activity is the primary means for controlling the level of cholesterol biosynthesis, which is the target of statin drugs $[19,20]$. Synthesized cholesterol and other dietary cholesterol is transported in the serum via LDLR-mediated uptake and HDL-mediated reverse transports [21], or catalyzed by CYP7A1 and then excreted in the bile $[22,23]$. The present study demonstrated that the treatment of hyperlipidemic hamsters with berberine, palmatine, coptisine, and TRCA obviously suppressed HMGCR expression. Moreover, in response to berberine, coptisine, jatrorrhizine, palmatine, epiberberine, and TRCA, hamsters exhibited a higher level of CYP7A1. Besides, berberine, jatrorrhizine, coptisine, TRCA, and palmatine enhanced the LDLR expression. The combined effects of the increased LDLR and CYP7A1 levels may account for the declined TC level in the therapy groups. Meanwhile, the increased bile acids and cholesterol excretions in the feces of hyperlipidemic hamsters suggest a possibility of inhibiting cholesterol and bile acid absorption and reabsorption. ASBT is another well-known therapeutic target for antihyperlipidemic drugs. ASBT inhibitors would increase excretion of bile acids, causing increased catabolism of hepatic cholesterol to bile acids. It is recognized that decreased FGF15 and FGF19 expression in mice ileal will lead to increases in hepatic CYP7A1 expression and fecal bile acids excretion $[24,25]$. Out et al. recently demonstrated that intestinal microbiota alters the Gata4-controlled expression of ASBT and affects the host bile acids reabsorption [26]. In light of these findings, it is important to investigate whether RC alkaloids regulate ASBT expression and determine the molecular mechanisms involved in the process. Kong et al. have recognized that berberine could activate the extracellular-regulated kinase pathway [9]. Since all RC alkaloids have the isoquinoline parent structure, it is rational for us to conclude that RC alkaloids also could activate the extracellular-regulated kinase pathway and increase LDLR expression. Mitochondrial uncoupling proteins are responsible for the generation of adenosine triphosphate (ATP) [27,28]. Among them, UCP-2 was recognized as a critical regulator of cellular fuel utilization and whole body glucose and lipid metabo- lism. A study involving 603 subjects has revealed that uncoupling protein 2 is associated with obesity [29]. The increased transcriptional activation of hepatic UCP-2 can effectively prevent body weight gain, adiposity, and lipid disorders [30]. It has been demonstrated that UCP mediates lipid metabolism via mitochondrial fatty acid efflux to increase fatty acid oxidation [31]. The present study demonstrated that berberine, palmatine, and TRCA can elevate the UCP-2 mRNA expression in hamsters, indicating that these agents could increase energy expenditure [32]. These results suggested that RC alkaloids and TRCA could retard the synthesis of cholesterol by downregulating HMGCR expression and accelerate the metabolism of lipids by upregulating LDLR, CYP7A1, and UCP-2 expression in the liver of hyperlipidemic hamsters.

To demonstrate which mechanism is responsible for the observed lipid changes, a correlation coefficient analysis between the TC level and the expression of HMGCR, CYP7A1, and LDLR was conducted. A negative correlation was found between the TC level and CYP7A1 content in hamsters $(r=-0.768, p=0.044)$. However, there was no significant correlation between the TC level and HMGCR and LDLR content ( $\mathrm{p}=0.06, \mathrm{p}=0.07$, respectively). This indicates that among these three lipid metabolism-related proteins, CYP7A1 plays a vital role in mediating the TC level. It is not surprising that coptisine, berberine, jatrorrhizine, palmatine, and epiberberine exhibited different antihyperlipidemic activities in the hamsters. Although these five alkaloids belong to the isoquinoline alkaloid family, they possess different substituent groups on the isoquinoline parent structure, and thus exhibit different pharmacological properties. Jung et al. have revealed that the presence of the methylenedioxy group in the D ring of epiberberine and groenlandicine was responsible for butyrylcholinesterase inhibition activity [33]. Pharmacokinetic studies have revealed that TRCA, berberine, and coptisine were mainly distributed in the liver of rats [34]. Among them, berberine transported more easily across the intestinal barrier of diabetic rats [35]. These findings may explain the excellent anti-hyperlipidemic activities of TRCA, berberine, and coptisine in this study. 

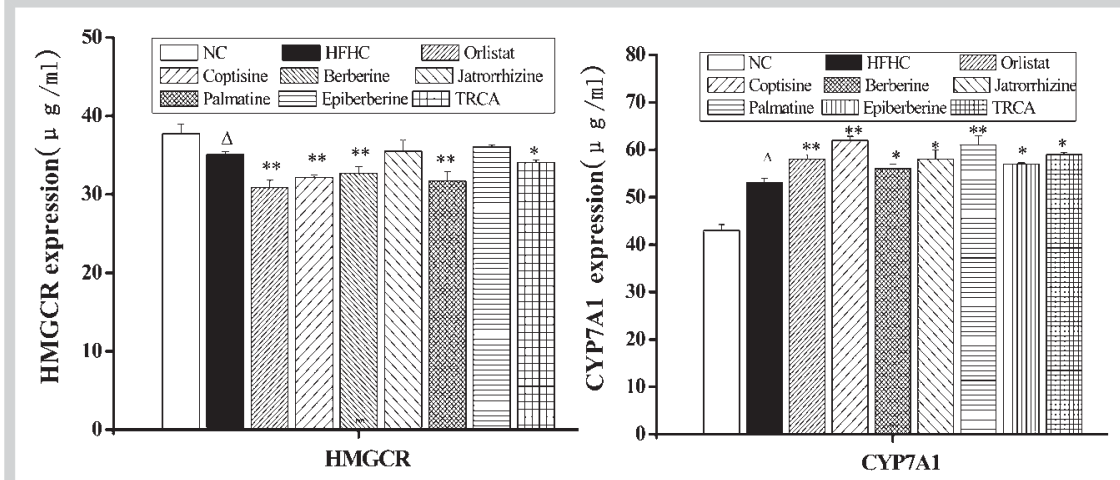

Fig. 5 ELISA analysis of cholesterol metabolismrelated protein in the livers of the hamsters. Orlistat, coptisine, berberine, palmatine, and TRCA significantly reduced the protein level of HMCCR. All pure RC alkaloids and TRCA significantly improved the expression of CYP7A1. Except for epiberberine, the LDLR protein levels were increased after RC alkaloid treatment. Values are means $\pm S D(n=3)$. ${ }^{\Delta} p<0.05,{ }^{\Delta \Delta} p<0.01$ vs. normal control; ${ }^{*} p<0.05$, ${ }^{* *} \mathrm{p}<0.01$ vs. HFHC.

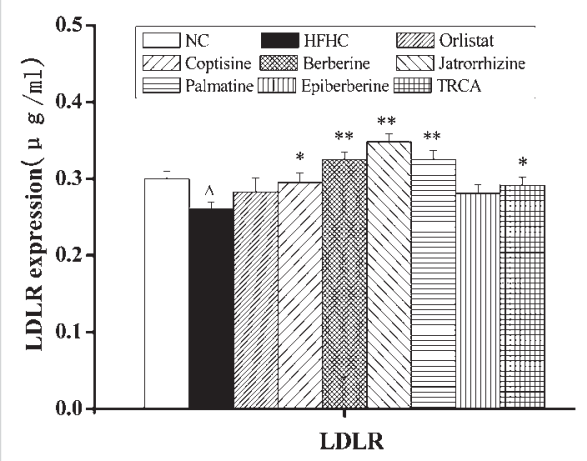

The use of a mono-compound purified from RC like berberine, palmatine, coptisine, jatrorrhizine, and epiberberine has proved to be a very powerful agent in modulating the numerous cellular signaling pathways including AMPK signaling [36], NF- $k B$ signaling [37], PPAR signaling [38], etc. Therefore, the five alkaloids in TRCA may regulate either the same or different targets in various lipids metabolism pathways. Thus, it is not surprising that TRCA extracts exhibited greatly amplified antihyperlipidemic effects when compared with pure RC alkaloids. Nevertheless, it is should be noted that other minor components in TRCA may also contribute to the lipid-lowering effect of TRCA. The precise and detailed mechanism of action for RC alkaloids on lipid metabolism remains to be elucidated.

Taken together, our results suggested that pure RC alkaloids and TRCA showed excellent lipid regulating activities and could reverse the body weight gain of hamsters induced by HFHC. These observed antihyperlipidemic effects can be attributed to the metabolism and excretion of cholesterol by RC alkaloids via downregulating the expression of HMGCR and upregulating the expression of LDLR and CYP7A1 as well as promoting the excretion of total bile acids in the feces. These findings highlight the critical role of RC alkaloids in hyperlipidemia treatment. Thus, they need to be considered in the future therapeutic approaches.

\section{Materials and Methods \\ $\nabla$}

\section{Plant materials and chemicals}

RC was obtained from Good Agricultural Practices Demonstration Base in Shizhu City and authenticated by Prof. LuJang Yuan at Southwest University. Coptisine, berberine, jatrorrhizine, palmatine, and epiberberine with a purity of 99.0\%, 99.3\%, 98.4\%, $98.9 \%, 98.8 \%$, respectively (by HPLC analysis) were separated from RC according to previous methods of our lab [39]. Briefly, $\mathrm{RC}$ ethanol extracts were subjected to high-speed counter cur- rent chromatography using a solvent system consisting of $\mathrm{CHCl} 3-\mathrm{MeOH}-0.2 \mathrm{M} \mathrm{HCl}(4: 2: 2, \mathrm{v} / \mathrm{v} / \mathrm{v})$. Peak fractions were collected according to the elution profiles. Then, the main fractions were further purified by a Sephadex LH-20 column to obtain the five main alkaloids. The spectrum data of these compounds are shown in Figs. $1 \mathrm{~S}$ to $5 \mathrm{~S}$, Supporting Information. Standard RC alkaloids were purchased from Chengdu Must Bio-technology Co., Ltd. and its purity was $>98 \%$ (batch number 14025939). Orlistat (99.9\%) was purchased from Zein Pharmaceutical. TC, TG, LDL-C, and HDL-C in the serum were estimated using commercially available kits. Elisa Commercial kits were purchased from Beijing Bioss Biotechnology Co., Ltd. All other chemicals used were of analytical grade.

\section{High-performance liquid chromatography analysis}

Chromatographic analysis of the RC alkaloids in the extract was performed on an LC-20 A series equipped with an SPD-M20 A photodiode array detector. The chromatographic separation was performed on a Hypersil ODS2 column $(250 \mathrm{~mm} \times 4.6 \mathrm{~mm}, 5 \mu \mathrm{m})$. The mobile phase consisted of an acetonitrile-water solution of $50 \mathrm{mmol} / \mathrm{L}$ potassium dihydrogen phosphate $(50: 50, \mathrm{v} / \mathrm{v}, \mathrm{pH} 4.0$, containing $15 \mathrm{mmol} / \mathrm{L}$ lauryl sodium sulfate). The column temperature was $30^{\circ} \mathrm{C}$. The flow rate was set at $0.6 \mathrm{~mL} / \mathrm{min}$. Ten $\mathrm{mg}$ of the total RC alkaloid extract was dissolved in $2 \mathrm{~mL}$ of methanol and then the solution was filtered with a $0.45-\mu \mathrm{M}$ membrane filter prior to HPLC analysis. The detection wavelength was set at $254 \mathrm{~nm}$ with a sample injection volume of $20 \mu \mathrm{L}$.

\section{Animals}

Healthy 5-week-old male Syrian golden hamsters (Mesocricetus auratus) weighing $100 \pm 10 \mathrm{~g}$ were obtained from Beijing Vital River Laboratory Animal Technology Co., Ltd. Animals were raised in stainless steel wire-mesh cages individually in a room kept at $22 \pm 2{ }^{\circ} \mathrm{C}$ with $50-60 \%$ relative humidity and a 12 -h light/dark cycle (light period: 8:00-20:00 h) and allowed free access to 
normal food and water. After acclimatization with the facility for 1 week, animals were randomly assigned to the normal or HFHC group. The HFHC diet was composed of $10 \%$ lard, $10 \%$ egg yolk powder, and $1 \%$ cholesterol. The normal diets were based on a modification of the recommendations of the American Institute of Nutrition.

After 8 weeks, hyperlipidemic hamsters were determined by the lipid levels including serum TC, TG, LDL-C, and HDL-C and randomly divided into HFHC, coptisine, berberine, jatrorrhizine, palmatine, epiberberine (with a dose of $46.7 \mathrm{mg} / \mathrm{kg} \times$ day), and TRCA groups (with a dose of $46.7 \mathrm{mg} / \mathrm{kg} \times$ day containing $11.0 \%$ jatrorrhizine, $8.0 \%$ epiberberine, $9.8 \%$ coptisine, $9.0 \%$ palmatine, and $47.0 \%$ berberine) and $1.2 \mathrm{mg} / \mathrm{kg} \times$ day of orlistat served as a positive control. Each group had six hamsters. In a subsequent experiment, except for the normal group, all hamsters were feed with HFHC diets. Hamsters were administered $0.7 \mathrm{~mL}$ of the corresponding drugs or saline (normal and HFHC groups) by gavage during the study period. Body weights were recorded every 7 days. Feces of each hamster were collected on the third day prior to termination of the study. After sacrification of the hamsters, the relative organ weights (\% ratio to final body weight) of selected vital organs were measured and subsequently kept in $10 \%$ neutral buffered formalin for pathological examination. This study was carried out in strict accordance with the recommendations in The Guide for the Care and Use of Laboratory Animals of the National Institutes of Health. All experimental protocols were approved in May 2012 by the Institutional Animal Ethics Committee of Southwest University (Permit Number: 12-1036).

\section{Observation for lipid metabolism \\ in Syrian golden hamsters}

TC, TG, LDL-C, and HDL-C were evaluated by commercially available kits (Huili Biotechnology) according to the manufacturer's instructions. Briefly, the TC concentration was determined by the cholesterol oxidase method, TG was determined by the glycerol-3-phosphate oxidase-p-aminophenazone method, and LDL-C concentrations were quantified by precipitation with phosphotungstic acid and $\mathrm{MgCl}_{2}$. HDL-C concentrations were quantified by the same method used to determine total cholesterol after the removal of other lipoproteins. The concentrations of TBA were measured enzymatically using the $3 \alpha$-HSD method [40] by a commercially available kit (Shanghai Rongsheng Biotechnology Technology).

Bioassays of total cholesterol and bile acids in the feces Feces of each hamster were collected 3 days prior to termination of the study for the analysis of cholesterol and bile acids. Feces powder $(0.5 \mathrm{~g})$ was extracted three times with $10 \mathrm{~mL}$ of $95 \%$ ethanol at $60^{\circ} \mathrm{C}[41]$. After being filtered, the supernatant was concentrated under reduced pressure, and the residue was dissolved in PBS to analyze the levels of TC and TBA.

\section{Quantitation of mRNA expression using quantitative reverse transcription-PCR}

Total RNA was prepared from livers using Trizol. cDNA was synthesized using SuperScript III First-Strand Synthesis System (Invitrogen). The qRT-PCR was performed on a Bio-Rad CFX connect real-time system. The sequences of the primers were as follows: HMGCR forward, 5'-TGG AGA TCA TGT GCT GCT TC-3', reverse, 5'GCG ACT ATG AGC GTG AAC AA-3'; LDLR forward, 5'-GAA AAG GCT ACT GGC TGT GC-3', reverse, 5'-CCA GGA CCC GGT CAG TAG TA-3'; CYP7A1 forward, 5'-ACA CCA TTC CTG CAA CCT TC-3', re- verse, 5'-GCT GTC CGG ATA TTC AAG GA-3'; UCP-2 forward, 5'GCG TTC TGG GTA CCA TCC TA-3', reverse, 5'-GCT CTG AGC CCT TGG TGT AG-3'; GAPDH forward, 5'-AAC TTT GGC ATT GTG GAA GG-3', reverse, 5'-ACA CAT TGG GGG TAG GAA CA-3'. The following cycling conditions were used: one denaturing cycle at $95^{\circ} \mathrm{C}$ for $3 \mathrm{~min}$, and 35 cycles of amplification $\left(95^{\circ} \mathrm{C}\right.$ for $10 \mathrm{~s}, 58^{\circ} \mathrm{C}$ for $10 \mathrm{~s}$, and $72^{\circ} \mathrm{C}$ for $30 \mathrm{~s}$ ) were performed followed by $5 \mathrm{~min}$ of final extention at $72^{\circ} \mathrm{C}$. Relative RNA levels were determined by analyzing the changes in SYBR Green fluorescence during PCR by the $2^{-\triangle \triangle C T}$ method according to the manufacturer's instructions. GAPDH was amplified in parallel for normalization [42]. The PCR products were confirmed by electrophoresis in $2 \%$ agarose gel stained with ethidium bromide. The purity of the amplified PCR products was determined by melting point analysis using ICycler software.

\section{Quantitation of protein expression using ELISA}

The livers of the hamsters were homogenized to detect the protein levels of HMGCR, LDLR, and CYP7A1 according to the manufacturers' instructions of ELISA kits (Biovalue) using a BioTek Elx 808 absorbance microplate reader.

\section{Statistical analysis}

Statistical analysis was performed by SPSS software for Windows (version 15.0). Student's t-test was calculated for comparison between normal and HFHC groups. Factorial significance of the interaction between lipid-lowering effects and body weights was assessed using an analysis of covariance (ANCOVA) with body weights as the covariate. Comparisons between treatment and control groups were performed by one-way or two-way analyses of variance (ANOVA) followed by post hoc Bonferroni's test, when appropriate. $P$ values $<0.05$ were considered significant.

\section{Supporting information}

Details on the body weight of the hamsters and spectral data of the compounds are available as Supporting Information.

\section{Acknowledgements \\ $\nabla$}

This work was financially supported by the High-End Engineering and Technical Personnel Training Plan of Chongqing (20132016), the Specialized Research Fund for the Doctoral Program of Higher Education (20130182 110023), the Achievement Transfer Program of Institutions of Higher Education in Chongqing (KJZH14203), The Special Program for Scientific and Technical Innovation of Chongqing Social Livelihood (cstc2015shmszx80012), and the Research Fund of Hunan Provincial Education Department (13B088).

\section{Conflict of Interest $\nabla$}

The authors declare no identifiable conflict of interest.

\section{References}

1 Yan W. Efficiency comparison of aerobic exercise combination of stomach muscle training in abdominal obesity individual. Chinese J Gerontol 2015; 2: 477-479

2 Genest J, Mcpherson R, Frohlich J, Anderson T, Campbell N, Carpentier A, Couture P, Dufour R, Fodor G, Francis GA, Grover S, Gupta M, Hegele RA, Lau DC, Leiter L, Lewis GF, Lonn E, Mancini GB, Ng D, Pearson GJ, Sniderman A, Stone JA, Ur E. 2009 Canadian Cardiovascular Society/Canadian 
guidelines for the diagnosis and treatment of dyslipidemia and prevention of cardiovascular disease in the adult - 2009 recommendations. Can J Cardiol 2009; 25: 567-579

3 Liu HH, Li JJ. Aging and dyslipidemia: a review of potential mechanisms. Ageing Res Rev 2015; 19: 43-52

4 Li ZY, Yang RF, Xu GB, Xia T. Serum lipid concentrations and prevalence of dyslipidemia in a large professional population in Beijing. Clin Chem 2005; 51: 144-150

5 Manickam P, Rathod A, Panaich S, Hari P, Veeranna V, Badheka A, Jacob S, Afonso L. Comparative prognostic utility of conventional and novel lipid parameters for cardiovascular disease risk prediction: do novel lipid parameters offer an advantage? J Clin Lipidol 2011; 5: 82-90

6 Ioannides-Demos LL, Piccenna L, Mcneil JJ. Pharmacotherapies for obesity: past, current, and future therapies. J Obes 2011; 2011: 179674

7 Group UDS. U.K. Prospective Diabetes Study 27. Plasma lipids and lipoproteins at diagnosis of NIDDM by age and sex. Diabetes Care 1997; 20: 1683-1687

8 Ikeda H, Taketomi S, Sugiyama Y, Shimura Y, Sohda T, Meguro K, Fujita T. Effects of pioglitazone on glucose and lipid metabolism in normal and insulin resistant animals. Arzneimittelforsch 1990; 40: 156-162

9 Kong W, Wei J, Abidi P, Lin M, Inaba S, Li C, Wang Y, Wang Z, Si S, Pan H, Wang S, Wu J, Wang Y, Li Z, Liu J, Jiang JD. Berberine is a novel cholesterol-lowering drug working through a unique mechanism distinct from statins. Nat Med 2004; 10: 1344-1351

$10 \mathrm{Hu}$ Y, Ehli EA, Kittelsrud J, Ronan PJ, Munger K, Downey T, Bohlen K, Callahan L, Munson V, Jahnke M, Marshall L, Nelson K, Huizenga P, Hansen $R$, Soundy T, Davies GE. Lipid-lowering effect of berberine in human subjects and rats. Phytomedicine 2012; 19: 861-867

11 Lee YS, Kim WS, Kim KH, Yoon MJ, Cho HJ, Shen Y, Ye JM, Lee CH, Oh WK, Kim CT, Behrens CH, Gosby A, Kraegen EW, James DE, Kim JB. Berberine, a natural plant product, activates AMP-activated protein kinase with beneficial metabolic effects in diabetic and insulin-resistant states. Diabetes 2006; 55: 2256-2264

12 Brusq JM, Ancellin N, Grondin P, Guillard R, Martin S, Saintillan Y, Issandou $M$. Inhibition of lipid synthesis through activation of AMP kinase: an additional mechanism for the hypolipidemic effects of berberine. J Lipid Res 2006; 47: 1281-1288

13 Hui D, Yan Z, Li Z, Fuer L. The effects of berberine on blood lipids: a systemic review and meta-analysis of randomized controlled trials. Planta Med 2013; 79: 437-446

14 Kahlon T, Chow F, Knuckles B, Chiu M. Cholesterol-lowering effects in hamsters of b-glucan-enriched barley fraction, dehulled whole barley, rice bran, and oat bran and their combinations. Cereal Chem 1993; 70: 435-440

15 Hokanson JE, Austin MA. Plasma triglyceride level is a risk factor for cardiovascular disease independent of high-density lipoprotein cholesterol level: a metaanalysis of population-based prospective studies. J Cardiovasc Risk 1996; 3: 213-219

16 Assmann G, Schulte H. Relation of high-density lipoprotein cholesterol and triglycerides to incidence of atherosclerotic coronary artery disease (the PROCAM experience). Am J Cardiol 1992; 70: 733-737

17 Brehm BJ, Seeley RJ, Daniels SR, D'Alessio DA. A randomized trial comparing a very low carbohydrate diet and a calorie-restricted low fat diet on body weight and cardiovascular risk factors in healthy women. J Clin Endocrinol Metab 2003; 88: 1617-1623

18 Bartness TJ, Wade GN. Photoperiodic control of seasonal body weight cycles in hamsters. Neurosci Biobehav Rev 1985; 9: 599-612

19 Haerer W, Delbaere K, Bartlett H, Lord S, Rowland J. Relationships between HMG-CoA reductase inhibitors (statin) use and strength, balance and falls in older people. Intern Med J 2012; 42: 1329-1334

20 Notarnicola M, Messa C, Refolo MG, Tutino V, Miccolis A, Caruso MG. Synergic effect of eicosapentaenoic acid and lovastatin on gene expression of HMGCoA reductase and LDL receptor in cultured HepG2 cells. Lipids Health Dis 2010; 9: 135-143

21 Han H, Xin P, Zhao L, Xu J, Xia Y, Yang X. Excess iodine and high-fat diet combination modulates lipid profile, thyroid hormone, and hepatic LDLr expression values in mice. Biol Trace Elem Res 2012; 147: 233239

22 Chen $Q$, Wang E, Ma L, Zhai P. Dietary resveratrol increases the expression of hepatic $7 \alpha$-hydroxylase and ameliorates hypercholesterolemia in high-fat fed C57BL/6 J mice. Lipids Health Dis 2012; 11: 56-63
23 Chiang JYL. Bile acids: regulation of synthesis. J Lipid Res 2009; 50: 1955-1966

24 Lan T, Rao A, Haywood J, Kock ND, Dawson PA. Mouse organic solute transporter alpha deficiency alters FGF15 expression and bile acid metabolism. J Hepatol 2012; 57: 359-365

25 Walters JR, Tasleem AM, Omer OS, Brydon WG, Dew T, le Roux CW. A new mechanism for bile acid diarrhea: defective feedback inhibition of bile acid biosynthesis. Clin Gastroenterol Hepatol 2009; 7: 1189-1194

26 Out C, Patankar JV, Doktorova M, Boesjes M, Bos T, de Boer S, Havinga $R$, Wolters $H$, Boverhof $R$, van Dijk TH, Smoczek A, Bleich A, Sachdev V, Kratky D, Kuipers F, Verkade HJ, Groen AK. Gut microbiota inhibit Asbtdependent intestinal bile acid reabsorption via Gata4. J Hepatol 2015; 63: 697-704

27 Divakaruni AS, Brand MD. The regulation and physiology of mitochondrial proton leak. Physiology (Bethesda) 2011; 26: 192-205

28 Mailloux RJ, Harper ME. Uncoupling proteins and the control of mitochondrial reactive oxygen species production. Free Radic Biol Med 2011; 51: 1106-1115

29 Oktavianthi S, Trimarsanto H, Febinia CA, Suastika K, Saraswati MR, Dwipayana P, Arindrarto W, Sudoyol H, Malik SG. Uncoupling protein 2 gene polymorphisms are associated with obesity. Cardiovasc Diabetol 2012; 11: 41-51

30 Oh K, Kim M, Lee J, Kim M, Nam Y, Ham J. Liver PPARalpha and UCP2 are involved in the regulation of obesity and lipid metabolism by swim training in genetically obese $\mathrm{db} / \mathrm{db}$ mice. Biochem Biophys Res Commun 2006; 345: 1232-1239

31 Harper ME, Dent R, Monemdjou S, Bézaire V, Van WL, Wells G, Kavaslar GN, Tesson F, McPherson R. Decreased mitochondrial proton leak and reduced expression of uncoupling protein 3 in skeletal muscle of obese diet-resistant women. Diabetes 2002; 51: 2459-2466

32 Faggioni R, Shigenaga J, Moser A, Feingold KR, Grunfeld C. Induction of UCP2 gene expression by LPS: a potential mechanism for increased thermogenesis during infection. Biochem Biophys Res Commun 1998; 244: 75-78

33 Jung HA, Min BS, Yokozawa T, Lee JH, Kim YS, Choi JS. Anti-Alzheimer and antioxidant activities of Coptidis Rhizoma alkaloids. Biol Pharm Bull 2009; 32: 1433-1438

34 Wang L, Ye X, Li X, Chen Z, Chen X, Gao Y, Zhao Z, Huang W, Chen X, Yi J. [Metabolism, transformation and distribution of Coptis chinensis total alkaloids in rat]. China J Chinese Materia Med (Zhongguo Zhong Yao Za Zhi) 2010; 35: 2017-2020

35 Yu S, Yu Y, Liu L, Wang X, Lu S, Liang Y, Liu X, Xie L, Wang G. Increased plasma exposures of five protoberberine alkaloids from Coptidis Rhizoma in streptozotocin-induced diabetic rats: is P-GP Involved? Planta Med 2010; 76: 876-881

36 Kim HS, Kim MJ, Kim EJ, Yang Y, Lee MS, Lim JS. Berberine-induced AMPK activation inhibits the metastatic potential of melanoma cells via reduction of ERK activity and COX-2 protein expression. Biochem Pharmacol 2012; 83: 385-394

37 Jiang $Q$ Liu P, Wu X, Liu W, Shen X, Lan T, Xu S, Peng J, Xie X, Huang H. Berberine attenuates lipopolysaccharide-induced extracelluar matrix accumulation and inflammation in rat mesangial cells: involvement of NF- $\kappa$ B signaling pathway. Mol Cell Endocrinol 2011; 331: 34-40

38 Sangeetha M, Priya C, Vasanthi HR. Anti-diabetic property of Tinospora cordifolia and its active compound is mediated through the expression of Glut-4 in L6 myotubes. Phytomedicine 2013; 20: 246-248

39 Chen HY, Ye XL, Cui XL, He K, Jin YN, Chen Z. Cytotoxicity and antihyperglycemic effect of minor constituents from Rhizoma Coptis in HepG2 cells. Fitoterapia 2012; 83: 67-73

40 Monte MJ, El-Mir MY, Sainz GR, Bravo P, Marin JG. Bile acid secretion during synchronized rat liver regeneration. Biochim Biophys Acta 1997; 1362: 56-66

41 Laura $C B$, Noemí $R$, Catherine $F$, Rosa R, Francisco BV, Joan C. Liver X receptor-mediated activation of reverse cholesterol transport from macrophages to feces in vivo requires ABCG5/G8. J Lipid Res 2008; 49: 904-911

42 Barber RD, Harmer DW, Coleman RA, Clark BJ. GAPDH as a housekeeping gene: analysis of GAPDH mRNA expression in a panel of 72 human tissues. Physiol Genomics 2005; 21: 389-395 